\title{
Frequency effects in language acquisition: a case study of plural forms in Brazilian Portuguese
}

Thais Cristófaro Silva ${ }^{1}$ and Christina Abreu Gomes ${ }^{2}$

${ }^{1}$ Department of Linguistics, Federal University of Minas Gerais, Brazil

${ }^{2}$ Department of Linguistics, Federal University of Rio de Janeiro, Brazil

https://doi.org/10.36505/ExLing-2008/02/0052/000111

\begin{abstract}
This paper discusses the acquisition of plural forms of words that end in a back glide in Brazilian Portuguese and may take two plural forms: either $-\mathrm{s}$ (the regular suffix) or the suffix -is. Fourty children were presented to picture cards containing words ending in a back glide and were asked to identify what they saw. Children's answers presented the suffix -is for the vast majority of words rather than the regular plural suffix $-\mathrm{s}$. Our results indicate that children abstract schemas rather than acquire default rules. The -is suffix has a very high frequency rate for words that end in a back glide which yields to the establishment of robust schemas. We argue that the plural suffix -is is adopted by children due to frequency effects which foments a very robust schema and enhances productivity.
\end{abstract}

Key-words: acquisition; plural, language use, frequency, schemas

\section{Morphological storage and language acquisition}

The study of the acquisition of regular and irregular inflection is an important issue in the debate as to how morphology is represented in the speaker's grammar/mind. More specifically, this debate addresses how children cope with the competing regular and irregular morphemes during the acquisition process. However, this last issue is interpreted according to the representational model adopted. This paper intends to be a contribution to such a debate by evaluating the acquisition of plural morphemes in Brazilian Portuguese.

In relation to representational models or models of morphological storage, two major trends can be identified: the dual and the single models. The differences between these models reflect different conceptions of how the speakers' grammars are organized. In the dual-processing model regular and irregular inflections differ structurally in terms of storage and processing (Marcus et. al., 1992, Prasada and Pinker, 1993, among others). Regular inflections are derived from a symbolic default rule while irregular inflected words are stored in the lexicon. Thus there is a structural difference between the storage and processing of regular and irregular morphological patterns.

In single-mechanism models, storage and processing of morphological forms are related to a single associative mechanism and regular or irregular forms are treated in the same way as separate entries stored in the lexicon.

Proceedings of the $2^{\text {nd }}$ ISCA Workshop on Experimental Linguistics, ExLing 2008, 25-27 August 2008, Athens, Greece. 
Thus, there is no difference in storage and processing of regular and irregular morphological patterns as stated in dual-processing model.

In connectionist models (Macwhinney and Leinbach, 1991) and the network model (Bybee, 1995), all types of morphological properties of words, paradigms and morphological patterns emerge from associations made among the words related in lexical representation. This model suggests that the lexicon is conceived as an organized net of lexical relations which interact in a network fashion. The regularities and similarities observed in linguistic items are properties that contribute to the structure of storage. This assumption is sustained by researches on lexical access. According to Bybee (1995), identity relations are established by phonetic and semantic similarities. Activation of one item spreads to other items. When words are related by parallel semantic and phonological connections, the resulting relations are morphological. In the single-mechanism model type and token frequency play a role in determining representation and type frequency is important in determining the regularity and productivity (Bybee, 1995). Overregularizations found in language acquisition process are interpreted as the overapplication of the most frequent pattern in the lexicon (Bybee, 1995).

This paper intends to show that the network model, which argues that frequency effects play a major role in the organization of Grammar, offers a general and elegant account for the acquisition of plural forms of words that end in a back glide in Brazilian Portuguese (henceforth BP).

\section{Inflection of words ending in a back glide in BP}

Words ending in a back glide may take two plural forms in BP: either $-\mathrm{s}$ (i.e., the regular plural suffix) or the suffix -is (which involves a vocalized lateral). Words which take the regular plural suffix - s present a back glide in any variety of Portuguese. For example, the singular form pau [paw] 'wood' has its plural form as [paws] 'wood(s)'. Words which take the plural suffix is present systematically a back glide in the singular forms for most varieties of $\mathrm{BP}^{1}$. For example, the singular for sal [saw] 'salt' has its plural form as [sais] 'salt(s)'. The back glide in this later case, i.e., sal [saw] 'salt', involves lateral vocalization which is already a completed change in the analyzed varieties of BP (Quednau, 1994). Lateral vocalization created then analogous segmental sequences in the end of words: a vowel followed by a back glide (pau [paw] 'wood' and sal [saw] 'salt'). The plural forms of words containing these word-final analogous vowel-glide segmental sequences are different: either an -s suffix, as for paus [paws] 'woods', or an -is suffix, as in sais [sais] 'salts', occurs. In this paper we will show that the choice between these plural suffixes during language acquisition follows from frequency generalizations over the lexicon. 


\section{Results and discussion}

Data were obtained in a controlled experiment with cards presented to the children who were requested to identify the pictures. A card containing a single object was presented to the child and the interviewer pronounced the word in its singular form. Then, immediately after another card was presented to the child with the same object illustrated more than once so that the child was expected to say the plural form. The data collection was recorded using a digital tape recorder. Only the answers with a plural morpheme were considered. The test consisted of 12 real words and 3 nonce words (total 15 words). The three nonce words were presented to participants in the singular form as being an imaginary toy or cartoon character. Token frequency and children's vocabulary were considered when selecting the words to be examined. Frequency count was obtained from a 130 million words corpus and grouped as high frequency and low frequency (www.projetoaspa.org). The 12 real words were grouped as either frequent or infrequent according to the corpus consulted. The audio containing the children's responses was verified by at least two researchers and submitted to statistical analysis of Minitab Program 13 for windows.

We wanted to test at what extend the default regular plural suffix $-\mathrm{s}$ would occur in words that ended in a back glide. If a default rule was acquired, as suggested by the dual mechanism model, one would expect that children would use preferably the suffix $-\mathrm{s}$ for words ending in a back glide. This is because the $-\mathrm{s}$ suffix is the regular plural marker for words ending in vowels and diphthongs in BP. However, this was not the case. In fact children presented the suffix -is for the vast majority of words which ended in a back glide (79\%). In words where the -is suffix was expected, as in sais [sais] 'salts', a rate of $94,86 \%$ was attested. In words where the - s suffix was expected, as in degraus [degraws] 'steps', we rather found the rate of $73,51 \%$ for the suffix -is. Thus, the -is irregular plural suffix occurred at a high rate $(73,51 \%)$ where the regular suffix $-\mathrm{s}$ was expected. We suggest that these results follow from frequency effects. Plural forms of words that end in a back glide count over a million tokens and 877 types for the -is suffix. Concerning the - s suffix the token count is just nearly 34.000 words and types count simply 33 words. This shows that the -is suffix occurs at a higher rate for token and type frequency when compared to the $-\mathrm{s}$ suffix. Pseudowords also took the irregular suffix -is at a higher rate than the regular suffix $-\mathrm{s}$. This indicates that the irregular suffix - is is preferred whether or not the word is known.

We suggest that our results indicate that children abstract schemas rather than acquire default rules, thus offering evidence for a network model (Bybee 1995). We argue that the plural marker -is is adopted by children due to its type frequency, an effect that foments a very robust schema and 
enhances productivity. The -is suffix has a very high type frequency rate for words that end in a back glide which yields to the establishment of robust schemas. That is why the suffix -is is preferred by children. Our results support the view that frequency effect shapes representation and also contributes towards the debate on the acquisition of irregular morphology (Marcus et al, 1992).

\section{Notes}

1. An alveolar lateral occurs in other varieties of Portuguese where the glide occurs in BP : [saw] alternates with [sal] 'salt', having both the plural: [sais].

\section{Acknowledgements}

CNPq/Brasil PQ grants 30.33.97/2005-5 and 304056/2007-3.

\section{References}

Bybee, Joan L. 1988. Morphology as lexical organization. In M. Hammond and M. Noonan (eds.) Theoretical morphology. Academic Press.119-141.

Bybee, Joan. 1995. Regular morphology and the lexicon. Cognitive Processes 10. 425-455.

MacWhinney, B. and Leinbach, J. 1991. Implementations are not conceptualizations: Revising the verb learning model.Cognition,29,121.

Marcus, G.F., Pinker, S., Ullman, M., Hollander, M., Rosen, T.J. and Xu, F. 1992. Overregularization in language acquisition. Monographs of the Society for Research in Child Development, 57 (4).

Prasada, S and Pinker, S. 1993. Generalisation of regular and irregular morphological patterns. Language and Cognitive Processes, 8, 1-56. 